\title{
Immediate Implant Reconstruction after Eliminating Polyacrylamide Hydrogel (Amazingel) in Complicated Breasts
}

\section{Jun-Young Jang, June-Kyu Kim}

Department of Plastic and Reconstructive Surgery, Kangbuk Samsung Hospital, Sungkyunkwan University School of Medicine, Seoul, Korea

No potential conflict of interest relevant to this article was reported.
Many injection materials have been used in cosmetic surgery for soft-tissue contour correction or breast augmentation. In China, Amazingel injection mammaplasty had been used widely because the procedure was non-invasive and easy to perform. But, numerous complications have been reported after injection of Amazingel composed of polyacrylamide gel such as breast pain, hardening, deformity, lump, gel migration, etc. In this article, we report a case of a 37-year-old Chinese female who complained of breast discomfort and deformity after Amazingel injection. We safely eliminated Amazingel and reconstructed the breasts with silicone implants. We could achieve aesthetically tolerable results.

Keywords Breast implants, Injections, Mammaplasty

\section{INTRODUCTION}

Augmentation mammaplasty is one of the most popular aesthetic operations in the world. With the rapid development of the Asian economy, the demand for breast augmentation has increased rapidly [1]. For simple breast augmentation, many attempts have been made to find a new and safe synthetic injectable material [2].

Amazingel (NanFeng Medical Science and Technology Development Co., Ltd., Shijiazhuang, People's Republic of China) is a semi-permanent filler composed of polyacrylamide hydrogel. As it can augment the breast simply through a syringe injection, it has been widely used in China since 1997. Since then, about 500,000 Chinese females have been injected with Amazingel. However, the use of this material has been banned since April 2006 due to its nu-

Received: Jan 12, 2016 Revised: Mar 19, 2016 Accepted: Mar 21, 2016 Correspondence: June-Kyu Kim Department of Plastic and Reconstructive Surgery, Kangbuk Samsung Hospital, Sungkyunkwan University School of Medicine, 29 Saemunan-ro, Jongno-gu, Seoul 03181, Korea.

E-mail: kokoro72@naver.com

Copyright @ 2016 The Korean Society for Aesthetic Plastic Surgery.

This is an Open Access article distributed under the terms of the Creative Commons Attribution Non-Commercial License (http://creativecommons.org/licenses/by-nc/4.0/) which permits unrestricted non-commercial use, distribution, and reproduction in any medium, provided the original work is properly cited. www.e-aaps.org merous side effects [1]. The purpose of this report is to describe a case of a patient who presented with complications after Amazingel injection.

\section{CASE REPORT}

A 37-year-old Chinese female patient visited our plastic surgery department with a chief complaint of breast deformity. She had received Amazingel injection in both breasts about 15 years previously from a non-medical practitioner. Her breasts gradually increased in size with deformity and irregularity. She had heard about the possibility of severe complications such as infection or necrosis, and hence, she presented to our clinic although she had only mild breast discomfort.

She had no underlying diseases or past medial history. On clinical examination, huge, soft, visible lumps were detected in the whole breast, including the upper and lower portion of the chest wall. The skin was irregular and thinned out, but it was soft without hardness. The lower pole skin under the nipple was markedly relaxed and drooped, causing pseudo-ptosis (Fig. 1).

A preoperative laboratory test and imaging study of the breasts were performed. The laboratory findings were normal. On magnetic resonance imaging (MRI), the Amazingel had a high signal intensity on T2-weighted images and low signal intensity on T1- 

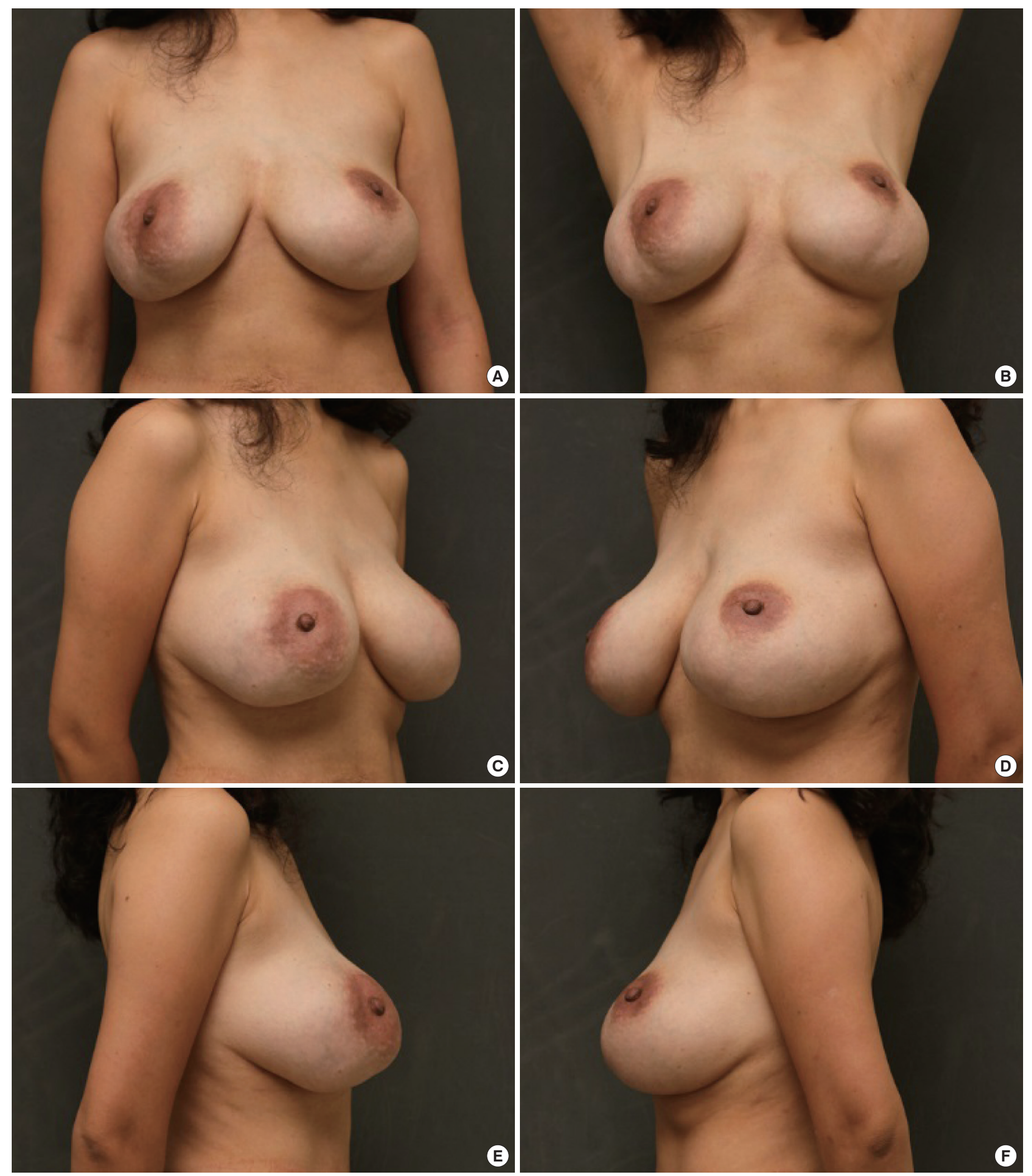

Fig. 1. Preoperative appearance. Pseudo-ptotic breast with asymmetry. Well-marginated lumps were observed in the inferior-medial areas of both breasts, which were noticeable when the patient raised her arm. 

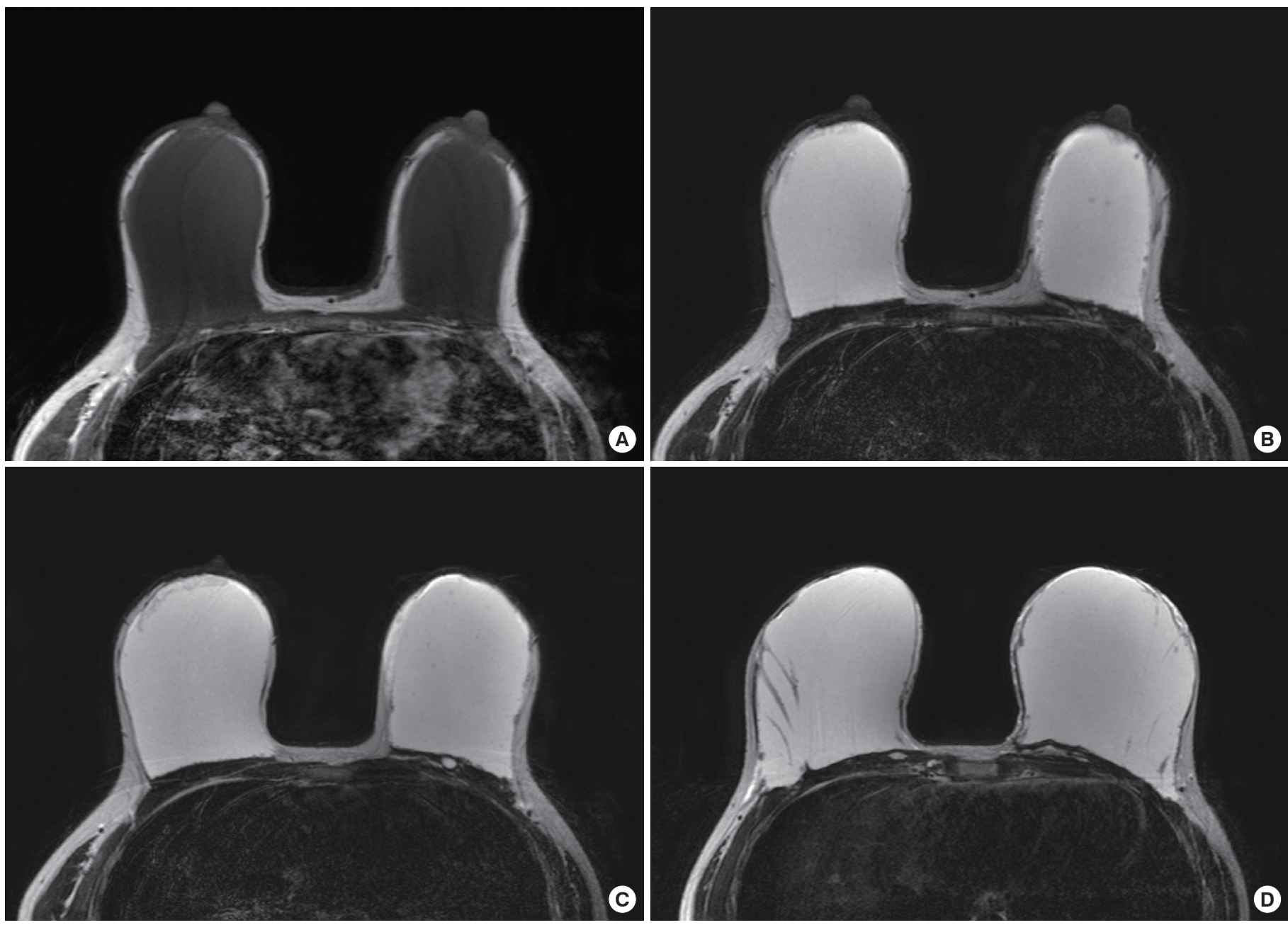

Fig. 2. Contrast-enhanced breast magnetic resonance (MR) images of Amazingel. Axial T1-weighted turbo spin-echo (repetition time [TR]/echo time $[T E]=7.2 / 3.7 \mathrm{~ms}$ ) and axial T2-weighted turbo spin-echo (TR/TE = 4850/124 ms) MR images were obtained. Amazingel showed hypo-intensity on T1-weighted images (A) and hyper-intensity on T2-weighted images (B-D). Most of the gel was encapsulated in the sub-glandular plane superficial to the pectoral muscles, and the glandular tissue had severely atrophied (A, B). Some portion of the gel was located in the left pectoralis major muscle (C) and the deep dermis of the breast skin (D).

weighted images. Breast MRI was performed with a 3.0 T MR scanner (Achieva, Philips Medical Systems, Best, Netherlands) with an 8 -channel breast coil. The breast was mostly occupied by polyacrylamide gel [3]. Most of the polyacrylamide gel was located in the sub-glandular plane within the capsule and the glandular tissue was severely atrophied as dermal fat. Some portion of the Amazingel had entered into the left medial breast skin and pectoralis major muscle (Fig. 2).

The patient wanted to undergo immediate breast reconstruction; hence, with her consent, we planned to remove most of the materials with the capsule and peripheral foreign body granuloma, and to conduct immediate breast reconstruction using silicone implants.

For inframammary fold repositioning, we designed a fusiform marking in the lower pole skin whose bottom line was the infra- mammary fold. Peri-areolar mastopexy was designed to achieve proper nipple position and to manage the remnant skin envelope (Fig. 3).

An incision was made on the upper border of the fusiform area after de-epithelization, in which the dermal portion acted as acellular dermal matrix to strengthen the lower pole skin. Through the window, we could identify thin fibrous capsules surrounding the Amazingel (Fig. 3). We could not eliminate them together because the capsules were very thin and could be easily ruptured. Yellow, granular gel was removed easily from the mammary area.

We then eliminated the capsule (Fig. 4). A palpable foreign body granuloma was also removed. The volume of the foreign body material was $420 \mathrm{cc}$ on the right side and $455 \mathrm{cc}$ on the left side (Fig. 5). Then, the cavities were thoroughly irrigated with dilute betadine solution and saline solution. After Barovac insertion into each breast, 


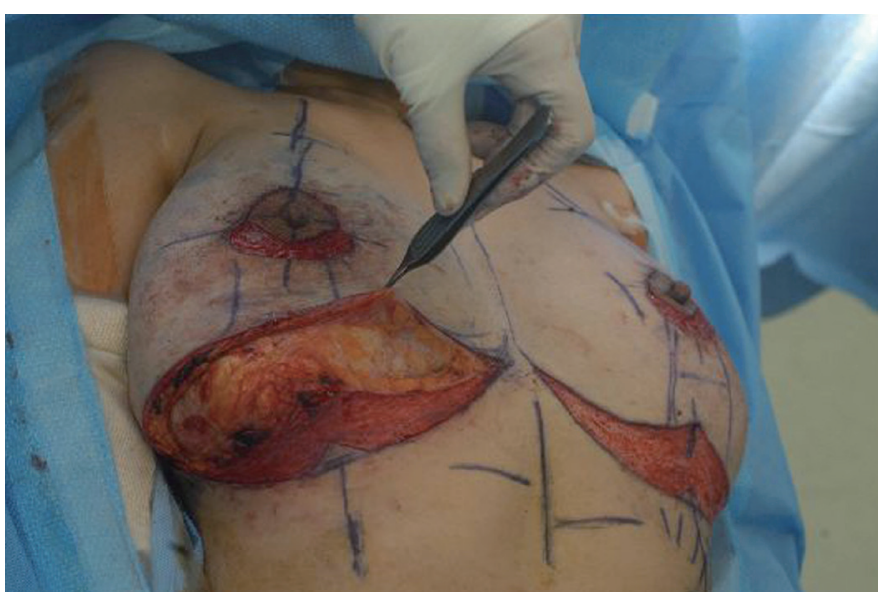

Fig. 3. Intra-operative picture. De-epithelization was performed below the nipple areolar complex and inframammary fold for repositioning. Through the inframammary fold incision, Amazingel, encapsulated within the fibrous tissue, was removed. A thin fibrous capsule was observed, and it was grasped with forceps.

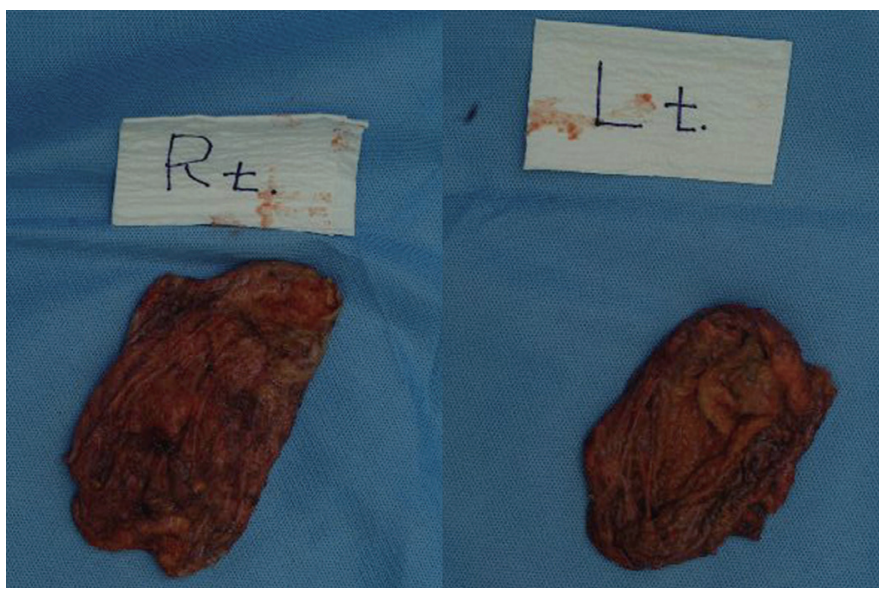

Fig. 4. Removal of fibrous capsular tissues. The resected fibrous capsule was very pulpy and easily torn.

we inserted $395 \mathrm{cc}$ silicone breast implants (Mentor, Santa Barbara, CA, USA) into the sub-glandular plane.

After surgery, we obtained a smooth contour with symmetry (Fig. 6). The pseudo-ptosis and deformity were corrected, and the breast discomfort disappeared. There were no complications such as infection, inflammation, or skin necrosis.

\section{DISCUSSION}

Amazingel is a permanent gel which consists of $5 \%$ polyacrylamide hydrogel polymer and $95 \%$ water [4].

It was first produced in Ukraine in the late 1980s under the name Interfall $[2,5,6]$. Injection of Amazingel offered a simple method for breast augmentation without invasive surgery. It was approved by

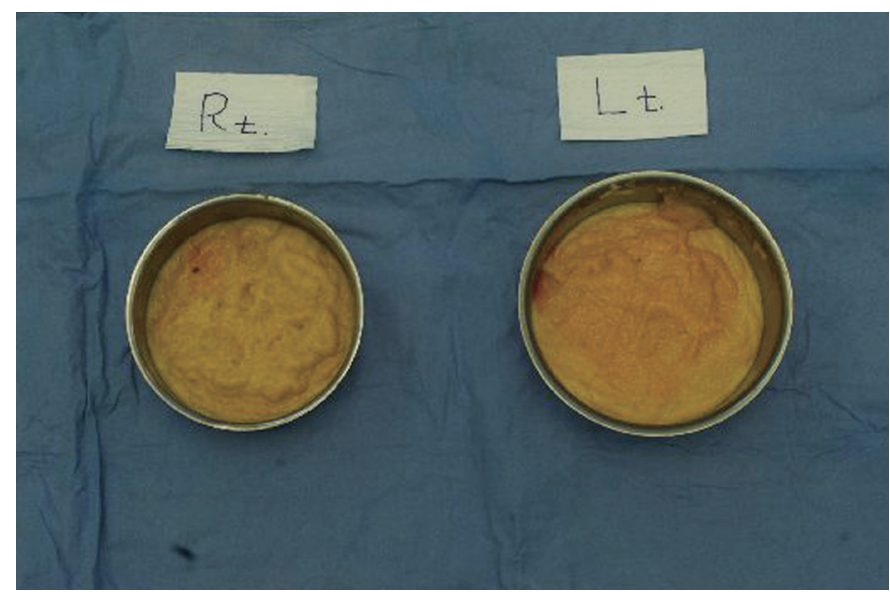

Fig. 5. Eliminated Amazingel. The Amazingel was a yellowish jellylike material. We eliminated $420 \mathrm{cc}$ from the right breast and $455 \mathrm{cc}$ from the left breast.

the State Drug Administration in China in 1997 [7-10], and many Chinese women received breast augmentation with Amazingel.

However, numerous complications were reported after Amazingel injection. Hence, its use was prohibited in most countries, including China [2]. Amazingel can cause irreversible tissue damage and many related complications [7-11]. The complications are as follows: pain (80\%), breast hardening (74\%), breast deformity (73\%), lumps (54\%), gel migration (37\%), fistula (16\%), and gel leakage (11\%) [2]. Also, severe complications like loss of lactation ability and breast cancer could be associated with Amazingel injection [12-14].

The removal of as much as possible of the Amazingel and its capsule is the only effective procedure to alleviate complications like breast pain, hardening, deformity, and lumps $[15,16]$. In 2012, Yu et al. [17] reported in their paper that the peri-areolar approach is superior for removal of Amazingel compared to the inframammary fold approach. They preferred the peri-areolar approach for the following reasons: First, Chinese patients usually refuse the inframammary fold approach. Second, it may be a convenient approach for gel that has migrated in the infra-clavicular region. Third, it is easy to dissect all the way up to the location of Amazingel. Finally, it is easy to squeeze the Amazingel from the breast.

In the patient described in this case report, most of the gel deposits were located over the pectoralis major muscle and its fascia within the fibrous capsule. Only a small amount of the Amazingel had infiltrated into the tissue, and there was no gel migration. It appears that the non-medical practitioner did not inject Amazingel evenly throughout the breast tissue; instead, the gel was injected solely in the sub-glandular plane. Hence, the patient only had a breast lump and deformity with mild discomfort. There were no complications such as gel leakage, skin necrosis, infection, or abscess formation. 

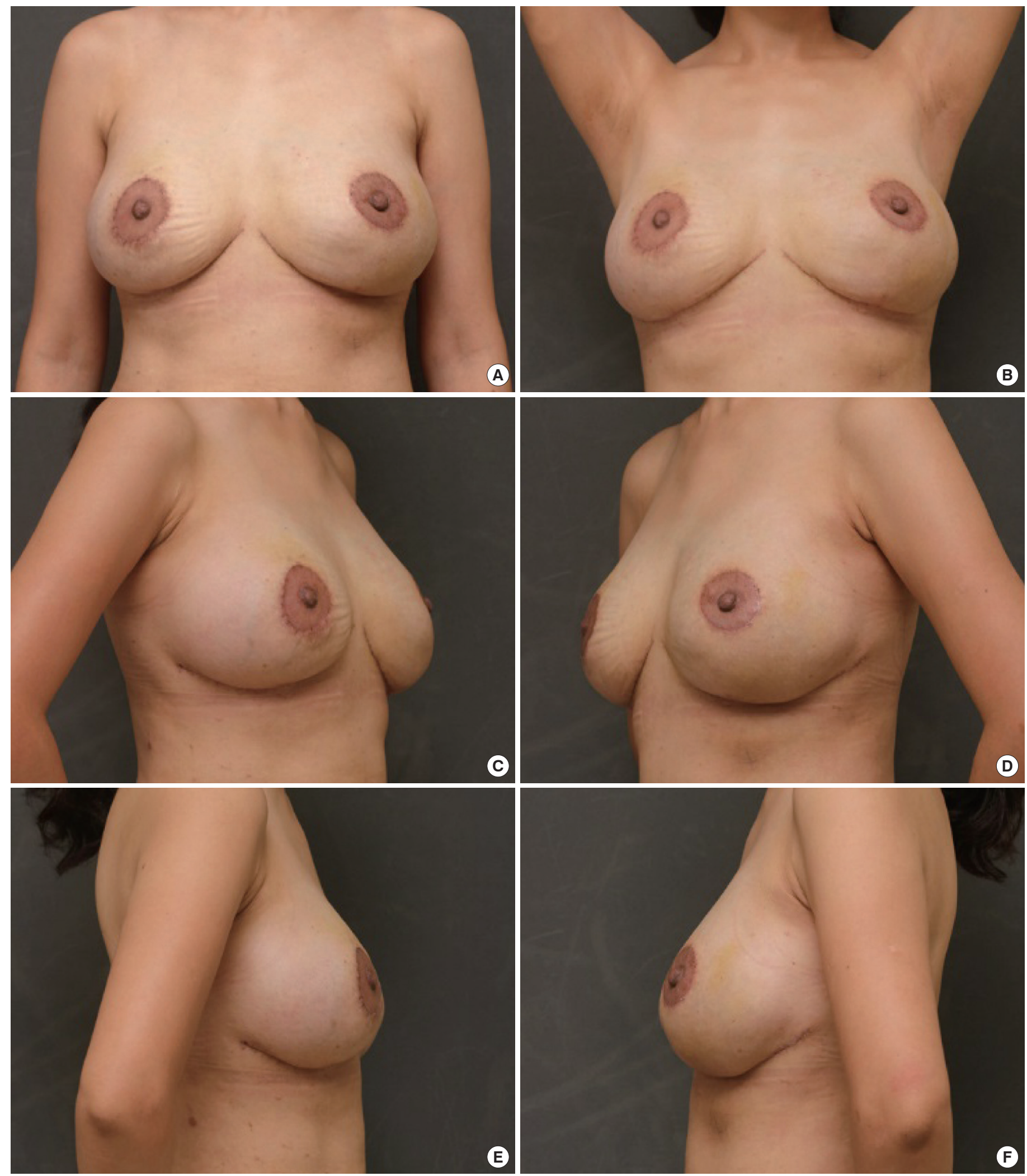

Fig. 6. Postoperative appearance. The final contour of the breast, six months after the insertion of a 395 cc Mentor anatomical implant. A symmetric, good aesthetic outcome was achieved. 
We chose to remove the Amazingel through the inframammary fold approach, and not the peri-areolar approach. There were several reasons for choosing this approach. First, the patient did not refuse the inframammary fold incision. Second, the Amazingel was well-encapsulated in the sub-glandular area with no migration, and it was possible to remove it sufficiently through the inframammary fold incision. Most importantly, we had to reconstruct the breast immediately.

We inserted synthetic implants in the sub-glandular plane, assuming that dissection of the pectoralis major muscles to insert the implants in the sub-muscular plane could have caused further spread of Amazingel-associated inflammation. Even though the breast tissue had atrophied, the skin envelope was preserved, which made immediate breast implant reconstruction possible. However, the thinned out skin over the lower pole of the breast needed to be reinforced. Hence, we deepithelialized the skin around the inframammary fold incision and used it as acellular dermal matrix to strengthen the lower pole skin. This is the reason why we chose the inframammary fold approach. After this, we inserted synthetic implants and performed inframammary fold repositioning and mastopexy.

For diagnosis, MRI is the most reliable tool to evaluate the nature and extent of foreign body invasion. Because Amazingel contains $95 \%$ water, it has high signal intensity on T2-weighted images and low signal intensity on T1-weighted images, like water $[3,18]$.

Amazingel can cause irreversible changes in the breast tissue in healthy women, requiring surgical intervention. If a patient has a history of receiving an Amazingel injection, preoperative imaging evaluation is necessary to assess its distribution and determine the appropriate surgical timing. We should eliminate as much of the gel as possible, and if remnant breast tissue is adequate for conducting reconstructive procedures like inframammary fold repositioning and mastopexy, implant insertion can be a useful technique to correct the deformity and restore a good breast contour.

\section{PATIENT CONSENT}

Patients provided written consent for the use of their images.

\section{REFERENCES}

1. Cheng MH, Huang JJ. Augmentation mammaplasty in asian women. Semin Plast Surg 2009;23:48-54.

2. Unukovych D, Khrapach V, Wickman M, et al. Polyacrylamide gel injections for breast augmentation: management of complications in 106 patients, a multicenter study. World J Surg 2012;36:695-701.

3. Lui CY, Ho CM, Iu PP, et al. Evaluation of MRI findings after polyacryl- amide gel injection for breast augmentation. AJR Am J Roentgenol 2008;191:677-88.

4. Andersen FA. Amended final report on the safety assessment of polyacrylamide and acrylamide residues in cosmetics. Int J Toxicol 2005; 24 Suppl 2:21-50.

5. Niechajev I. Lip enhancement: surgical alternatives and histologic aspects. Plast Reconstr Surg 2000;105:1173-83; discussion 84-7.

6. Christensen L, Breiting V, Janssen M, et al. Adverse reactions to injectable soft tissue permanent fillers. Aesthetic Plast Surg 2005;29:34-48.

7. Qiao Q, Wang X, Sun J, et al. Management for postoperative complications of breast augmentation by injected polyacrylamide hydrogel. Aesthetic Plast Surg 2005;29:156-61; discussion 62.

8. Cheng NX, Wang YL, Wang JH, et al. Complications of breast augmentation with injected hydrophilic polyacrylamide gel. Aesthetic Plast Surg 2002;26:375-82.

9. Niedzielska I, Pajak J, Drugacz J. Late complications after polyacrylamide hydrogel injection into facial soft tissues. Aesthetic Plast Surg 2006;30:377-8.

10. Cheng NX, Xu SL, Deng H, et al. Migration of implants: a problem with injectable polyacrylamide gel in aesthetic plastic surgery. Aesthetic Plast Surg 2006;30:215-25.

11. Christensen L, Breiting V. Management of postoperative complications of breast augmentation by injected polyacrylamide hydrogel. Aesthetic Plast Surg 2006;30:132.

12. Zhao Y, Yuan NA, Li K, et al. Bilateral breast cancer following augmentation mammaplasty with polyacrylamide hydrogel injection: a case report. Oncol Lett 2015;9:2687-93.

13. Cheng NX, Liu LG, Hui L, et al. Breast cancer following augmentation mammaplasty with polyacrylamide hydrogel (PAAG) injection. Aesthetic Plast Surg 2009;33:563-9.

14. Wang ZX, Luo DL, Dai X, et al. Polyacrylamide hydrogel injection for augmentation mammaplasty: loss of ability for breastfeeding. Ann Plast Surg 2012;69:123-8.

15. Luo SK, Chen GP, Sun ZS, et al. Our strategy in complication management of augmentation mammaplasty with polyacrylamide hydrogel injection in 235 patients. J Plast Reconstr Aesthet Surg 2011;64:731-7.

16. Ono S, Ogawa R, Hyakusoku H. Complications after polyacrylamide hydrogel injection for soft-tissue augmentation. Plast Reconstr Surg 2010;126:1349-57.

17. Yu L, Wang J, Zhang B, et al. Treatment of breast injection with polyacrylamide hydrogel with infiltrated fascia capsule removal: report on 104 cases. Aesthetic Plast Surg 2012;36:1120-7.

18. Khedher NB, David J, Trop I, et al. Imaging findings of breast augmentation with injected hydrophilic polyacrylamide gel: patient reports and literature review. Eur J Radiol 2011;78:104-11. 